\title{
Antibiotics and the resistant microbiome
}

\author{
Sommer, Morten; Dantas, Gautam
}

Published in:

Current Opinion in Microbiology

Link to article, DOI:

10.1016/j.mib.2011.07.005

Publication date:

2011

Link back to DTU Orbit

Citation (APA):

Sommer, M., \& Dantas, G. (2011). Antibiotics and the resistant microbiome. Current Opinion in Microbiology, 14(5), 556-563. https://doi.org/10.1016/j.mib.2011.07.005

General rights

Copyright and moral rights for the publications made accessible in the public portal are retained by the authors and/or other copyright owners and it is a condition of accessing publications that users recognise and abide by the legal requirements associated with these rights.

- Users may download and print one copy of any publication from the public portal for the purpose of private study or research.

- You may not further distribute the material or use it for any profit-making activity or commercial gain

- You may freely distribute the URL identifying the publication in the public portal

If you believe that this document breaches copyright please contact us providing details, and we will remove access to the work immediately and investigate your claim. 


\section{Elsevier Editorial System(tm) for Current Opinion in Microbiology Manuscript Draft}

Manuscript Number:

Title: Antibiotics and the resistant microbiome

Article Type: 14/5 Antimicrobials

Corresponding Author: Prof. Morten Otto Alexander Sommer, PhD

Corresponding Author's Institution: Technical University of Denmark

First Author: Morten Otto Alexander Sommer, PhD

Order of Authors: Morten Otto Alexander Sommer, PhD; Gautam Dantas, PhD 
TITLE: Antibiotics and the resistant microbiome

AUTHORS:

Morten O.A. Sommer $(1,2)$ and Gautam Dantas $(3,4)$

(1) Department of Systems Biology, Technical University of Denmark, DK2800 Lyngby, Denmark

(2) NNF Center for Biosustainability, Technical University of Denmark, DK2800 Lyngby, Denmark

(3) Center for Genome Sciences \& Systems Biology, Washington University School of Medicine, St Louis, MO, USA.

(4) Department of Pathology \& Immunology, Washington University School of Medicine, St Louis, MO, USA.

Correspondence should be addressed to msom@bio.dtu.dk (MOAS) and dantas@wustl.edu (GD)

\section{GLOSSARY:}

Human microbiota - the collection of microbes living in and on the human body Human microbiome - the collective genomic content of the human microbiota Resistome - the complement of all antibiotic resistance genes encoded by a microbial community

Metagenomic functional selection - a culture-independent method for capture of selectable functions from microbial metagenomic DNA through heterologous expression in an indicator host strain.

\section{SUMMARY:}

Since the discovery and clinical application of antibiotics, pathogens and the human microbiota have faced a near continuous exposure to these selective agents. A well-established consequence of this exposure is the evolution of multidrug-resistant pathogens which can become virtually untreatable. Less appreciated are the concomitant changes in the human microbiome in response to these assaults and their contribution to clinical resistance problems. Studies have shown that pervasive changes to the human microbiota result from antibiotic treatment and that resistant strains can persist for years. Additionally, culture independent functional characterization of the resistance genes from the microbiome has demonstrated a close evolutionary relationship between resistance genes in the microbiome and in pathogens. Application of these techniques and novel cultivation methods are expected to significantly expand our understanding of the interplay between antibiotics and the microbiome. 


\section{INTRODUCTION:}

Antibiotic compounds work to either stop bacteria from growing (bacteriostatic agents) or to kill them outright (bacteriocidal agents). The effectiveness of these compounds "against life" stems from their ability to block critical bacterial cellular processes [1|2]. Since these cellular targets are often highly genetically and structurally conserved across diverse members of the bacterial kingdom, deployment of an antibiotic compound against a specific bacterium (e.g. a pathogen) is quite likely to impart collateral damage to the bacterial community which shares the environment of the intended target organism. The spectrum of unintended effects of antibiotic therapy is likely to be considerably larger than simple growth inhibition of susceptible strains, especially considering the role of these bioactive organic compounds as multi-activity signaling molecules [3]. Almost every surface of the human body after birth is colonized by a rich and diverse community of commensal microbes [4-6], and this microbiota has substantial and continuous effects on human health and physiological development, including dietary and nutritional processing, prevention of pathogen invasion, and immune system maturation [7-9]. Accordingly, antibiotic treatment to prevent or eradicate a pathogenic infection at virtually any body site is likely to produce both short-term and long-term impacts on the commensal microbiota and its encoded microbiome [10]. These effects can be broadly categorized into two interrelated categories(Figure 1): (1) ones which change the relative proportions of different species in the microbiota, with introduction of a new species or complete eradication of an existing species as extreme scenarios $111-$ 16], and (2) ones which alter the antibiotic resistome encoded by members of the microbiota [17 [18]. In the first case, changes in the architecture of the microbiota can dramatically perturb the carefully evolved homeostasis of microbiota-host mutualism, which can lead to both transient and persistent changes in host physiology and health [19/20]. In the latter case, enrichment and exchange of resistance genes within the microbiota increase the accessibility of these elements to potentially pathogenic organisms, challenging our ability to treat their subsequent infections [21|22]. 


\section{MAIN TEXT:}

\section{Antibiotic induced changes in microbial community structure}

Dramatic advances over the past few years in next-generation DNA sequencing technology have enabled a series of recent high-resolution molecular characterizations of the change in microbiota architecture in humans and model organisms in response to antibiotic treatment. Deep 16S rDNA pyrosequencing was used to investigate the effect of two courses of ciprofloxacin on the gastrointestinal microbiota of three healthy individuals, which revealed dramatic and immediate antibiotic-induced decreases in phylogenetic diversity of the previously stable microbial ecosystem [12 15 . While the microbiota began to recover to a relatively stable state within a week after antibiotic insult, the pretreatment architecture had not fully recovered within 6-10 months, including the apparent complete loss of low-abundance members of the community. Similarly, a combination of $16 \mathrm{~S}$ sequencing and terminal-restriction fragment length polymorphism (T-RFLP) was used to measure the short- and long-term effects of clarithromycin and metronidazole, which are standard treatment for Helicobacter pylori eradication, on the throat and gastrointestinal microbiota of three treated human subjects compared to three controls [16]. Like the ciprofloxacin study, the combination antibiotic treatment caused transient decreases in microbiota diversity in both the throat and the gut, and the overall community architecture had only partially recovered one and four years after treatment. Interestingly, the throat microbiota was found to be more stable after antibiotic treatment and over long periods than the intestinal microbiota.

One of the challenges in fully understanding the mechanisms of antibioticinduced microbiota perturbations is the inherent baseline variability of the microbiota of different human individuals, which is confounded by the small sample size employed in human studies to date. One approach to address this variation is to use animal models, where host genotype, diet and other variables, which may impact baseline microbiota composition can be controlled, while still employing modern high-resolution molecular methods to characterize the microbiota [13/14]. A major finding of such studies is that different antibiotic regimens cause reproducible distinct short-term and long-term impacts on the microbiota in murine models. Mice treated with a combination of amoxicillin, metronidazole and bismuth had significant changes in abundance and structure of their cecal microbiota following treatment, but there was also significant rapid recovery of the community structure towards baseline after removal of the treatment. In contrast, treatment with the broad-spectrum antibiotic cefoperazone caused persistent depression in overall community diversity up to six weeks after treatment withdrawal, though community composition was 'normalized' when the treated cohort was co-housed with a non-treated animal, presumably via coprophagy [14]. In another experiment, mice treated with the glycopeptide vancomycin, which exclusively targets gram-positive organisms, showed similar effects to the combination treatment, where the antibiotic-induced community 
perturbations recovered after removal of treatment but differed from both the previous treatments in that vancomycin did not result in a major decrease in overall bacterial biomass [13. Of note, all treatments caused transient increases in the normally low-abundance Proteobacteria at the expense of the normally dominant Firmicutes and Bacteroidetes, which is particularly intriguing given the recent rapid global proliferation of extensively drug resistant strains of Protoeobacterial human pathogens, including Klebsiella pneumoniae, Acinetobacter baumanii, Pseudomonas aeruginosa, and extended-spectrum beta-lactamase producing Enterobacteriaceae 23.

The simplest mechanistic explanation for short-term antibiotic-induced changes in the microbiota is differences in the activity spectrum of the drug; drug resistant or insensitive bacteria proportionally increase at the expense of susceptible ones. For instance, the relative enrichment of the Proteobacteria and Tenericutes in mice cecal microbiota in response to Vancomycin is rationalized by innate resistance to this drug by these groups [13]. Vancomycin targets a peptidoglycan precursor needed for normal cell-wall synthesis, but is ineffective against these bacteria due to its inability to penetrate the Proteobacterial outer membrane, and the lack of a cell wall in Tenericutes comprised of peptidoglycan. However, antibiotics can also perturb the complex architecture of the microbiota through indirect or multilayered effects; for example, bacteria which are insensitive to direct action of an antibiotic might nevertheless still be affected if other bacteria or environmental factors which they are dependent on are changed by antibiotic treatment [19]. For instance, mice treated with the broad-spectrum combination metronidazole, neomycin and vancomycin significantly down-regulated intestinal expression of Regllly, a secreted C-type lectin which is induced by commensal microbes and kills gram-negative bacteria [20]. This down-regulation of the host innate immune defense in turn significantly decreased in vivo killing of vancomycin-resistant enterococci, demonstrating the indirect effects of antibiotic treatment on a pathogenic microbe.

\section{Persistent effects on the antibiotic resistome following antibiotic treatment}

A major expectation from repeated antibiotic exposure of the microbiota is an increasing abundance of antibiotic resistance determinants, caused by the growth advantage of resistant organisms during antibiotic treatment and further exacerbated through the lateral exchange of resistance determinants between diverse bacteria. Growth of this resistant population and their associated resistance genes is obviously dependent on the existence of a basal level of resistance in the microbiota; however, even in absence of direct anthropogenic treatment with antibiotics such populations are generally found present in human, animal and even 'pristine' environmental microbiota [24]. Studies using anaerobic and aerobic cultivation methods have shown that the oral microbiota of children that have not received antibiotics contain a subpopulation of different species that are resistant to tetracycline and other antibiotics [25]. Aerobic 
cultivation of enterobacteria from the gut of human volunteers free of antibiotics for over one year documented mean levels of resistance in excess of $20 \%$ to 13 different antibiotics [17]. Antibiotic treatment is expected to selectively increase the abundance of these organisms, leading to increased prevalence of their resistance genes and hence increasing the likelihood of their dissemination via lateral gene transfer. Indeed, a cultivation-based study documented significant increases in amoxicillin resistance levels in children exposed to this antibiotic in the three months after exposure, compared to a non-exposed control group [26].

It has long been hypothesized that resistance elements confer a fitness disadvantage to the host, and hence resistant strains should be outcompeted by susceptible counterparts once antibiotic pressure is removed [27]. Unfortunately, there is a growing appreciation that antibiotic resistance can persist for long periods in absence of treatment, as highlighted by the basal levels of resistance in the microbiota described above. Cultivation based studies have shown that treatment with clarithromycin leads to high level resistance within intestinal Enterococcus sp. during treatment and that the highly resistant Enteroccocus $s p$. persisted in several patients for 1-3 years after treatment ended [28. Similar results have been obtained for Staphyllococcus epidermis isolated from the nostrils of antibiotic treated patients [29] and Bacteriodes sp. isolated from the gut [30]. Furthermore, a culture independent PCR assay showed that the abundance of specific macrolide resistance genes had increased up to 10,000 fold compared to pre-treatment levels, and persisted up to two years after cessation of antibiotic treatment [31].

Mechanisms underlying the persistence of antibiotic resistance are complex and include compensatory adaptations and co-selection [32-35]. An elegant demonstration of how compensatory mutations lead to a reduced fitness cost associated with antibiotic resistance gene carriage was shown for two lineages of Bacteroides thetaiotaomicron from the human gut microbiota [36]. In individuals exposed to clindamycin over seven days, a dominant clone of $B$.

thetaiotaomicron acquired the erm $\mathrm{G}$ or $\mathrm{F}$ resistance gene, initially leading to a fitness cost in absence of antibiotic selection. However, resistant clones from this lineage isolated two weeks later had significantly reduced fitness cost associated with the carriage of this resistance gene, highlighting the rapid adaptations that can occur in vivo [36. Interestingly studies on the persistence of antibiotic resistance after cessation of antibiotic therapy in livestock microbiota document more drastic reductions in the prevalence of antibiotic resistance compared to those shown in the human microbiota [37]. The larger reductions in antibiotic resistance levels in livestock microbiota may result from the turnover of the sampled individuals; yet even under these conditions of rapid turnover, antibiotic resistance genes are expected to persist in life stock microbiota for decades after abolishment of antibiotic use [32].

\section{Transfer of antibiotic resistance determinants}


The dissemination of antibiotic resistance genes necessitates the physical transfer or acquisition of the genetic element encoding antibiotic resistance. The mechanisms responsible for this transfer are conjugation, transformation or transduction, all of which are fully capable of transferring antibiotic resistance determinants in vitro from resistant donor organisms to susceptible recipient organisms [21]38]. Such transfer events have been documented for strains isolated from virtually any reservoir in vitro under laboratory conditions. More complex in vitro systems mimicking the biofilm community structure of the oral microbiota have also been used to demonstrate transfer of antibiotic resistance genes between distant genera through both conjugation as well as transformation [39]. In vitro fermentation systems simulating the human intestinal system have also been used for the investigation of the horizontal transfer of extended spectrum beta-lactamases. While transfer of resistance genes was accelerated greatly by the administration of cefotaxime to which the donor strain harbored resistance, a base line of transconjugants were present shortly after introduction of the resistant donor strain even in absence of antibiotic selection [40.

To extend these in vitro findings, a number of groups have studied the in vivo transfer of antibiotic resistance genes in animal models. Germ free mice colonized with enterococcal donor and recipient strains were used to demonstrate the conjugal transfer of vancomycin resistance with appreciable rates and resulting in a stable population of transconjugants [41]. However, transfer occurred at much lower rates in mice colonized with the complex microbiota from feces recovered from healthy human subjects. Indeed transfer events were only documented between strains of the same species and these transconjugants did not stably colonize the microbiota [41]. The strong preferential transfer of vancomycin resistance to closely related strains was also recently confirmed for a larger array of clinical gram positive isolates in vitro [42]. Recently, transfer of the extended spectrum beta lactamase CTX-M-9 was shown in rats colonized with human microbiota between strains of Salmonella enterica and Escherichia coli; however, the transconjugants were again only transiently present in the rat microbiome and only during treatment with selective antibiotics [43].

Notably, a series of experiments have directly investigated the transfer of antibiotic resistance genes within the gut microbiota of healthy human subjects. In one study healthy human volunteers ingested cultures of Enterococcus faecium resistant to either glycopeptide or streptogramin antibiotics. Fecal samples from the individuals were sampled using cultivation-based methods and enterococcal isolates resistant to virginiamycin or vancomycin were enumerated. It was found that the ingested resistant strains were able to multiply and persist in the human gut environment for 7 days; however, no transfer events were detected as all resistant isolates were identical to the ingested strains based on the pulsed field gel electrophoresis pattern [22]. These experiments were 
followed up with a study demonstrating the in vivo transfer of the vanA resistance determinant from a donor Enterococcus faecium strain of animal origin to a recipient Enterococcus faecium strain of human origin in the intestine of healthy human subjects [44]. Similar results were shown for the sulfonamide resistance gene sul2 within strains of E. coli in the human intestine [45].

The in vivo studies on antibiotic resistance transfer highlight the importance not only of the transfer event but also the subsequent ability of the strain to establish within the microbiota. While there is a growing body of research documenting persistent effects on the microbiome community structure and the resistance levels within the microbiota, there is limited support yet for this from controlled in vivo studies in both animal and human microbiota. This apparent discrepancy may result from the somewhat artificial conditions of controlled in vivo studies where donor and recipient strains from a different microbiota are introduced to an already established microbiota. The stability of the established microbiota and the foreign origin of donor and recipient strains may be an important factor explaining the contrasting results. Another consequence of focusing the attention on predefined donor and recipient strains in controlled in vivo experiments so far is that the dissemination of antibiotic resistance genes within the total microbial community is not quantified.

\section{Microbiome wide characterization of the antibiotic resistome}

Previous culture based studies have thus clearly established that the human commensal microbiota harbors many multidrug resistant isolates, whose load of antibiotic resistance genes have increased steadily over the past decades. However, the majority of constituents in the microbiota have remained inaccessible to traditional culturing techniques, and molecular methods have estimated the gastrointestinal tract is home to nearly 1000 different species and over 7000 different strains [4\|10], indicating our picture of the antibiotic resistome of the human microbiota from culturing-studies is incomplete. In collaboration with Gordon and colleagues, we (Dantas) recently demonstrated that use of strict anaerobic conditions and a specially formulated rich gut microbiota medium enabled culturing of over $70 \%$ of genus level OTUs observed in corresponding uncultured "complete" microbiota [46]. While this is undoubtedly a dramatic improvement over past estimates of microbiota "culturability", the need for culture-independent methods for complete characterization of the resistome remains acute.

The most commonly used culture-independent method for detection and quantification of antibiotic resistance genes in polymicrobial samples is PCR with primers homologous to known resistance gene sequences [47] (Fig 2B). For example, quantitative PCR (qPCR) screens were recently used to quantify the levels of tet and erm genes, conferring resistance to tetracycline and macrolide, lincosamide, and streptogramin B antibiotics respectively, in animal and human 
waste water [48, highlighting potential routes of dissemination of resistance genes between human, animal and environmental microbiota. However, the extraordinary specificity of PCR based studies is also an important limitation of the technique: because PCR can only be used to interrogate a sample for known genes, it is an ineffective method for identifying novel resistance genes.

A complementary culture-independent method which enables characterization of both known and novel resistance genes is termed 'metagenomic functional selection' (Fig 2B), wherein total community microbial (metagenomic) DNA is extracted from any arbitrary source (e.g. a fecal microbiota sample) and cloned into an antibiotic susceptible indicator strain (e.g. E. coli) [17|47]. Indicator strains transformed with DNA that encode resistance genes can be functionally selected by plating the recombinant library on antibiotic media. Functionally selected metagenomic DNA inserts are amplified and sequenced to identify the captured resistance genes. We use the phrase metagenomic functional selections to specifically refer to the idea of subjecting metagenomic DNA to an experimental functional assay, generally through shot-gun expression in a heterologous host [49], in an attempt to distinguish this approach from broader definitions of "functional metagenomics", which include sophisticated computational approaches for annotation of functions in shot-gun metagenomic sequence data, but without direct experimental validation [50]. As with PCR-based screens, metagenomic functional selections were first developed and applied for resistome characterization of environmental microbial communities [24 477], and have subsequently been successfully applied to identify a variety of enzymatic functions from cultured and uncultured microbes, including genes and pathways for degradation of or resistance towards numerous xenobiotics [51|52].

We have used metagenomic functional selections to demonstrate that the resistome of the human gut microbiota had been profoundly under-sampled using traditional culture-based methods [17/49]. We identified several hundred antibiotic resistance genes within the microbial communities of two healthy, unrelated adults. Over $95 \%$ of the resistance genes in cultured Proteobacterial isolates from these microbiota were highly similar to previously characterized genes (>90\% nucleotide identity), many of which had been identified in pathogens. In sharp contrast, application of the same method to directly interrogate uncultured microbiota from the same fecal samples yielded predominantly novel resistance genes, with low sequence identity to previously characterized genes ( $<61 \%$ nucleotide identity). Furthermore, while over $65 \%$ of the resistance genes derived from cultured isolates were highly similar between the two individuals, $<10 \%$ of the resistance genes from culture-independent sampling were similar between these two individuals. Our results exposed the substantial under-sampling of resistance genes in prior studies of human microbiota, which stemmed from a reliance on culture-dependent methods. Importantly, our data underscore the utility of metagenomic functional selections for resistance gene discovery and obtaining a culture-unbiased depiction of the diverse and personalized resistomes of the microbiota in humans. 
With Gordon and co-workers, we (Dantas) recently showed that inter-individual differences in human microbiota antibiotic resistomes could be detected by subjecting both uncultured 'complete' fecal microbiota as well as pools of phylogenetically-representative fecal culture collections to metagenomic functional selections [46]. Notably, the metagenomic detection of specific resistance genes (e.g. encoding amikacin resistance) in complete samples significantly correlated with the fraction of sample-matched cultured isolates phenotypically resistant to those compounds, and the exact genes were then reconfirmed to be present in those cultured isolates by PCR. Additionally, when transplanted into gnotobiotic mice, complete and cultured communities exhibited similar colonization dynamics, biogeographical distribution, and responses to dietary perturbations, and these personalized culture collections could be enriched in vivo for taxa suited to specific perturbations. These methods for careful recapitulation and manipulation of microbiota both in vitro and in vivo hold great promise for quantifying the phylogenetic specificity of specific resistance genes and interrogating correlations between prevalence and dynamics of antibiotic resistomes and specific genetic and environmental variables.

\section{CONCLUSION:}

Antibiotic treatment leads to pervasive changes in the microbial community structure and persistent increases in antibiotic resistance amongst sampled cultivatable species. This results from the rapid proliferation of antibiotic resistant strains present in low basal levels in the microbiota and through horizontal transfer of antibiotic resistance genes. While advances in sequencing technology have driven increases in our knowledge of the effects of antibiotic treatment on total community architecture, we still have a limited understanding of the evolutionary pathways through which the genetic elements encoding antibiotic resistance spreads within a community. However, the application of metagenomic functional selections, novel cultivation methods and high throughput sequencing on microbiota exposed to antibiotics should enable a more detailed understanding of the processes governing the interplay between antibiotics and the resistant microbiome and its contribution to clinical problems with antibiotic resistance. 


\section{ACKNOWLEDGEMENTS:}

We thank H.J. Genee and Dr. A.M. Moore for assistance in creating figures.

Work in M.O.A.S's lab is supported by grants from the Novo Nordisk Foundation, Lundbeckfonden and EU FP7 Health program EvoTAR project. Work in G.D.'s lab is supported by the National Academies Keck Futures Initiative, the Washington University/Pfizer Biomedical Research Program, the Children's Discovery Institute and the International Center for Advanced Renewable Energy and Sustainability. 


\section{FIGURE LEGENDS}

Figure 1: The effects of antibiotic treatment on the microbiota. (A) Prior to antibiotic selection the constituents of the microbiota range in sensitivities towards antibiotics from sensitive (blue, green and yellow) to resistant (red). During exposure susceptible organisms will decrease in abundance, unless they acquire resistance determinants from viable resistant organisms. Upon cessation of treatment some of the susceptible organisms may increase in abundance to previous levels, whereas others will be lost from the microbiota. (B) During antibiotic exposure the microbial community architecture is significantly altered due to the differing sensitivities of the constituent microbes. Following cessation of antibiotic treatment community architecture largely re-establishes yet certain changes persist. (C) Schematic depiction of the changes in species diversity and antibiotic resistance levels upon antibiotic treatment followed by partial recovery of microbial community structure and resistance levels upon cessation of treatment.

Figure 2: Schematic representation of methods for characterizing antibiotic resistance from human fecal microbiota. (A) The traditional method for characterizing antibiotic resistant isolates from the fecal microbiota begins with phenotypic growth assays of individual cultured clones on antibiotic-containing media to determine the minimum inhibitory concentration (MIC). Resistance genes are detected by either PCR amplification based on the sequence of known genes or by whole genome sequencing followed by computational annotation of resistance genes using homology to known genes. (B) Culture-independent resistome approaches enable microbiota-wide characterization of resistance genes. Detection and quantification of known resistance genes can be accomplished by PCR. Characterization of both known and novel resistance genes can be accomplished through metagenomic functional selections, wherein metagenomic DNA is directly extracted from the fecal sample and cloned into an expression system in a cultivable, genetically-tractable host strain (e.g. E. coli). Metagenomic transformants harboring DNA fragments that encode antibiotic resistance genes are selected by subjecting the library of clones to specific antibiotics at concentrations which inhibit the growth of the untransformed indicator strain. Selected DNA fragments can then be sequenced to identify the specific resistance genes. 


\section{REFERENCES:}

1. Walsh C: Antibiotics : actions, origins, resistance. Washington, D.C.: ASM Press; 2003.

2. Kohanski MA, Dwyer DJ, Collins JJ: How antibiotics kill bacteria: from targets to networks. Nat Rev Microbiol 2010, 8:423-435.

3. Yim G, Wang HH, Davies J: Antibiotics as signalling molecules. Philos Trans $R$ Soc Lond B Biol Sci 2007, 362:1195-1200.

4. Nelson KE, Weinstock GM, Highlander SK, Worley KC, Creasy HH, Wortman JR, Rusch DB, Mitreva M, Sodergren E, Chinwalla AT, et al.: A catalog of reference genomes from the human microbiome. Science 2010, 328:994999.

5. Dominguez-Bello MG, Costello EK, Contreras M, Magris M, Hidalgo G, Fierer N, Knight R: Delivery mode shapes the acquisition and structure of the initial microbiota across multiple body habitats in newborns. Proc Natl Acad Sci U S A 2010, 107:11971-11975.

6. Qin J, Li R, Raes J, Arumugam M, Burgdorf KS, Manichanh C, Nielsen T, Pons N, Levenez F, Yamada T, et al.: A human gut microbial gene catalogue established by metagenomic sequencing. Nature 464:59-65.

7. Round JL, Mazmanian SK: The gut microbiota shapes intestinal immune responses during health and disease. Nat Rev Immunol 2009, 9:313-323.

8. Khoruts A, Dicksved J, Jansson JK, Sadowsky MJ: Changes in the composition of the human fecal microbiome after bacteriotherapy for recurrent Clostridium difficile-associated diarrhea. J Clin Gastroenterol 2010, 44:354-360.

9. Muegge BD, Kuczynski J, Knights D, Clemente JC, Gonzalez A, Fontana L, Henrissat $B$, Knight $R$, Gordon JI: Diet drives convergence in gut microbiome functions across mammalian phylogeny and within humans. Science 2011, 332:970-974.

10. Jernberg C, Lofmark S, Edlund C, Jansson JK: Long-term impacts of antibiotic exposure on the human intestinal microbiota. Microbiology 2010, 156:3216-3223.

11. Blaser MJ, Falkow $S$ : What are the consequences of the disappearing human microbiota? Nat Rev Microbiol 2009, 7:887-894.

12. Dethlefsen L, Relman DA: Incomplete recovery and individualized responses of the human distal gut microbiota to repeated antibiotic perturbation. Proc Natl Acad Sci U S A 2011, 108 Suppl 1:4554-4561.

13. Robinson CJ, Young VB: Antibiotic administration alters the community structure of the gastrointestinal micobiota. Gut Microbes 2010, 1:279-284.

14. Antonopoulos DA, Huse SM, Morrison HG, Schmidt TM, Sogin ML, Young VB:

Reproducible community dynamics of the gastrointestinal microbiota following antibiotic perturbation. Infect Immun 2009, 77:2367-2375.

$\left({ }^{*}\right)$ The authors use massively-parallel 16S rRNA sequencing to demonstrate that that different antibiotic regimens cause reproducible distinct short-term and longterm impacts on the phylogenetic architecture of mouse microbiota. 
15. Dethlefsen L, Huse S, Sogin ML, Relman DA: The pervasive effects of an antibiotic on the human gut microbiota, as revealed by deep $16 \mathrm{~S}$ rRNA sequencing. PLoS Biol 2008, 6:e280.

$\left(^{* *}\right)$ Deep 16S rDNA pyrosequencing revealed dramatic ciprofloxacin-induced decreases in phylogenetic diversity of the gastrointestinal microbiota of three healthy humans, and persistent changes were observed up to 6 months after treatment. First high-resolution culture-independent analysis of antibiotic changes in the human microbiota.

16. Jakobsson HE, Jernberg C, Andersson AF, Sjolund-Karlsson M, Jansson JK, Engstrand L: Short-term antibiotic treatment has differing long-term impacts on the human throat and gut microbiome. PLoS One 2010, 5:e9836.

17. Sommer MO, Dantas G, Church GM: Functional characterization of the antibiotic resistance reservoir in the human microflora. Science 2009, 325:1128-1131.

$\left.{ }^{* *}\right)$ Metagenomic functional selections reveal the massively undersampled antibiotic resistome of the uncultured human microbiota, while also showing that aerobic cultured microbiota isolates share resistance genes with human pathogens. First culture-independent functional characterization of resistance genes in human samples.

18. Shoemaker NB, Vlamakis H, Hayes K, Salyers AA: Evidence for extensive resistance gene transfer among Bacteroides spp. and among Bacteroides and other genera in the human colon. Appl Environ Microbiol 2001, 67:561-568.

19. Willing BP, Russell SL, Finlay BB: Shifting the balance: antibiotic effects on host-microbiota mutualism. Nat Rev Microbiol 2011, 9:233-243.

$\left.{ }^{* *}\right)$ Excellent review of how antibiotics perturb the evolved homeostasis of hostmicrobiota mutualism.

20. Brandl K, Plitas G, Mihu CN, Ubeda C, Jia T, Fleisher M, Schnabl B, DeMatteo RP, Pamer EG: Vancomycin-resistant enterococci exploit antibiotic-induced innate immune deficits. Nature 2008, 455:804-807.

$\left({ }^{* *}\right)$ Broad-spectrum combination antibiotic treatment in mice induced downregulation of Reglll $\gamma$, in turn significantly decreasing in vivo killing of antibioticresistant enterococci. First paper linking reduced innate immune defense due to antibiotic treatment to increased susceptibility to infection. 
21. Vignaroli C, Zandri G, Aquilanti L, Pasquaroli S, Biavasco F: Multidrug-resistant enterococci in animal meat and faeces and co-transfer of resistance from an Enterococcus durans to a human Enterococcus faecium. Curr Microbiol 2011, 62:1438-1447.

22. Sorensen TL, Blom M, Monnet DL, Frimodt-Moller N, Poulsen RL, Espersen F: Transient intestinal carriage after ingestion of antibiotic-resistant Enterococcus faecium from chicken and pork. $N$ Engl J Med 2001, 345:1161-1166.

23. Boucher HW, Talbot GH, Bradley JS, Edwards JE, Gilbert D, Rice LB, Scheld M, Spellberg B, Bartlett J: Bad bugs, no drugs: no ESKAPE! An update from the Infectious Diseases Society of America. Clin Infect Dis 2009, 48:1-12.

24. Allen HK, Donato J, Wang HH, Cloud-Hansen KA, Davies J, Handelsman J: Call of the wild: antibiotic resistance genes in natural environments. Nat Rev Microbiol 2010, 8:251-259.

25. Lancaster H, Ready D, Mullany P, Spratt D, Bedi R, Wilson M: Prevalence and identification of tetracycline-resistant oral bacteria in children not receiving antibiotic therapy. FEMS Microbiol Lett 2003, 228:99-104.

26. Ready D, Lancaster H, Qureshi F, Bedi R, Mullany P, Wilson M: Effect of amoxicillin use on oral microbiota in young children. Antimicrob Agents Chemother 2004, 48:2883-2887.

27. Andersson DI, Hughes D: Antibiotic resistance and its cost: is it possible to reverse resistance? Nat Rev Microbiol 2009, 8:260-271.

28. Sjolund M, Wreiber K, Andersson DI, Blaser MJ, Engstrand L: Long-term persistence of resistant Enterococcus species after antibiotics to eradicate Helicobacter pylori. Ann Intern Med 2003, 139:483-487.

29. Sjolund M, Tano E, Blaser MJ, Andersson DI, Engstrand L: Persistence of resistant Staphylococcus epidermidis after single course of clarithromycin. Emerg Infect Dis 2005, 11:1389-1393.

30. Lofmark S, Jernberg C, Jansson JK, Edlund C: Clindamycin-induced enrichment and long-term persistence of resistant Bacteroides spp. and resistance genes. J Antimicrob Chemother 2006, 58:1160-1167.

31. Jernberg C, Lofmark S, Edlund C, Jansson JK: Long-term ecological impacts of antibiotic administration on the human intestinal microbiota. Isme J 2007, 1:56-66.

32. Johnsen PJ, Townsend JP, Bohn T, Simonsen GS, Sundsfjord A, Nielsen KM: Retrospective evidence for a biological cost of vancomycin resistance determinants in the absence of glycopeptide selective pressures. J Antimicrob Chemother 2011, 66:608-610.

$\left.{ }^{*}\right)$ Elegant model for predicting the fitness cost of antibiotic resistance gene carriage and time scale for eventual clearance from microbial community

33. Johnsen PJ, Townsend JP, Bohn T, Simonsen GS, Sundsfjord A, Nielsen KM: Factors affecting the reversal of antimicrobial-drug resistance. Lancet Infect Dis 2009, 9:357-364. 
34. Andersson DI, Hughes D: Antibiotic resistance and its cost: is it possible to reverse resistance? Nat Rev Microbiol 2010, 8:260-271.

35. zur Wiesch PA, Kouyos R, Engelstadter J, Regoes RR, Bonhoeffer S: Population biological principles of drug-resistance evolution in infectious diseases. Lancet Infect Dis 2011, 11:236-247.

36. Lofmark S, Jernberg C, Billstrom H, Andersson DI, Edlund C: Restored fitness leads to long-term persistence of resistant Bacteroides strains in the human intestine. Anaerobe 2008, 14:157-160.

$\left({ }^{* *}\right)$ Seminal paper documenting in the human microbiota the acquisition of an antibiotic resistance gene upon antibiotic treatment following by the restoration of the fitness defect associated with resistance gene carriage.

37. Aarestrup FM, Seyfarth AM, Emborg HD, Pedersen K, Hendriksen RS, Bager F: Effect of abolishment of the use of antimicrobial agents for growth promotion on occurrence of antimicrobial resistance in fecal enterococci from food animals in Denmark. Antimicrob Agents Chemother 2001, 45:2054-2059.

38. Mazaheri Nezhad Fard R, Barton MD, Heuzenroeder MW: Bacteriophagemediated transduction of antibiotic resistance in enterococci. Lett Appl Microbiol 2011, 52:559-564.

39. Hannan S, Ready D, Jasni AS, Rogers M, Pratten J, Roberts AP: Transfer of antibiotic resistance by transformation with eDNA within oral biofilms. FEMS Immunol Med Microbiol 2010, 59:345-349.

40. Smet A, Rasschaert G, Martel A, Persoons D, Dewulf J, Butaye P, Catry B, Haesebrouck F, Herman L, Heyndrickx M: In situ ESBL conjugation from avian to human Escherichia coli during cefotaxime administration. $J$ Appl Microbiol 2011, 110:541-549.

41. Bourgeois-Nicolaos N, Moubareck C, Mangeney N, Butel MJ, Doucet-Populaire F: Comparative study of vanA gene transfer from Enterococcus faecium to Enterococcus faecalis and to Enterococcus faecium in the intestine of mice. FEMS Microbiol Lett 2006, 254:27-33.

42. Werner G, Freitas AR, Coque TM, Sollid JE, Lester C, Hammerum AM, GarciaMigura L, Jensen LB, Francia MV, Witte W, et al.: Host range of enterococcal vanA plasmids among Gram-positive intestinal bacteria. J Antimicrob Chemother 2011, 66:273-282.

43. Faure S, Perrin-Guyomard A, Delmas JM, Chatre P, Laurentie M: Transfer of plasmid-mediated CTX-M-9 from Salmonella enterica serotype Virchow to Enterobacteriaceae in human flora-associated rats treated with cefixime. Antimicrob Agents Chemother 2010, 54:164-169.

44. Lester CH, Frimodt-Moller N, Sorensen TL, Monnet DL, Hammerum AM: In vivo transfer of the vanA resistance gene from an Enterococcus faecium isolate of animal origin to an E. faecium isolate of human origin in the intestines of human volunteers. Antimicrob Agents Chemother 2006, 50:596-599. 
$\left.{ }^{*}\right)$ In vivo demonstration of transfer of antibiotic resistance genes in the intestinal microbiota of healthy human subjects.

45. Trobos M, Lester CH, Olsen JE, Frimodt-Moller N, Hammerum AM: Natural transfer of sulphonamide and ampicillin resistance between Escherichia coli residing in the human intestine. J Antimicrob Chemother 2009, 63:80-86.

46. Goodman AL, Kallstrom G, Faith JJ, Reyes A, Moore A, Dantas G, Gordon JI: Extensive personal human gut microbiota culture collections characterized and manipulated in gnotobiotic mice. Proc Natl Acad Sci $U$ $S$ A 2011, 108:6252-6257.

$\left({ }^{* *}\right)$ High-throughput anaerobic culturing techniques are coupled with gnotobiotic animal husbandry and metagenomics to show that the human fecal microbiota consists largely of taxa and predicted functions that are represented in its readily cultured members. Metagenomic functional selections were used to detect interindividual differences in the resistomes of both uncultured 'complete' fecal microbiota as well as pools of phylogenetically-representative fecal culture collections.

47. Handelsman J, Rondon MR, Brady SF, Clardy J, Goodman RM: Molecular biological access to the chemistry of unknown soil microbes: a new frontier for natural products. Chem Biol 1998, 5:R245-249.

48. Chen J, Michel FC, Jr., Sreevatsan S, Morrison M, Yu Z: Occurrence and persistence of erythromycin resistance genes (erm) and tetracycline resistance genes (tet) in waste treatment systems on swine farms. Microb Ecol 2010, 60:479-486.

49. Sommer MO, Church GM, Dantas G: The human microbiome harbors a diverse reservoir of antibiotic resistance genes. Virulence 2010, 1:299-303.

50. Dinsdale EA, Edwards RA, Hall D, Angly F, Breitbart M, Brulc JM, Furlan M, Desnues C, Haynes M, Li L, et al.: Functional metagenomic profiling of nine biomes. Nature 2008, 452:629-632.

51. Uchiyama T, Miyazaki K: Functional metagenomics for enzyme discovery: challenges to efficient screening. Curr Opin Biotechnol 2009, 20:616-622.

52. Sommer MO, Church GM, Dantas G: A functional metagenomic approach for expanding the synthetic biology toolbox for biomass conversion. $\mathrm{Mol}$ Syst Biol 2010, 6:360. 
Highlights:

1) Evolution of antibiotic resistance in pathogens has become a global public health threat

2) Microbiome wide characterization of resistance genes supports recent genetic exchange between the resistant microbiota and pathogens

3) Treatment with antibiotics leads to pervasive changes in the composition of the human microbiota and its encoded resistome

4) In vivo studies have demonstrated transfer of antibiotic resistance genes within the microbiota even in absence of exposure

5) Enrichment of resistance genes in the microbiota is likely to exacerbate problems with pathogenic resistance 
A

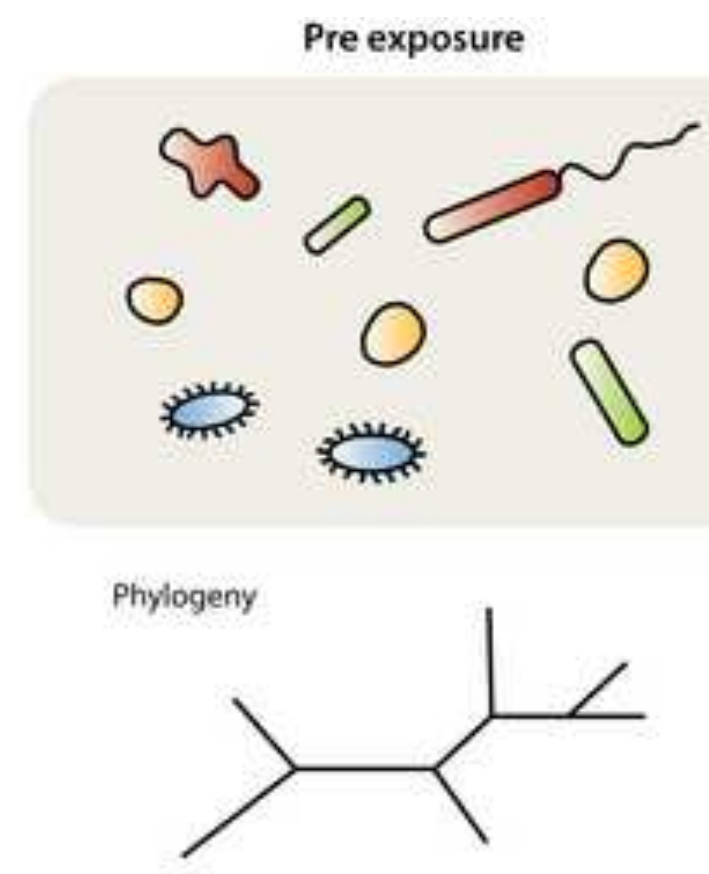

Exposed
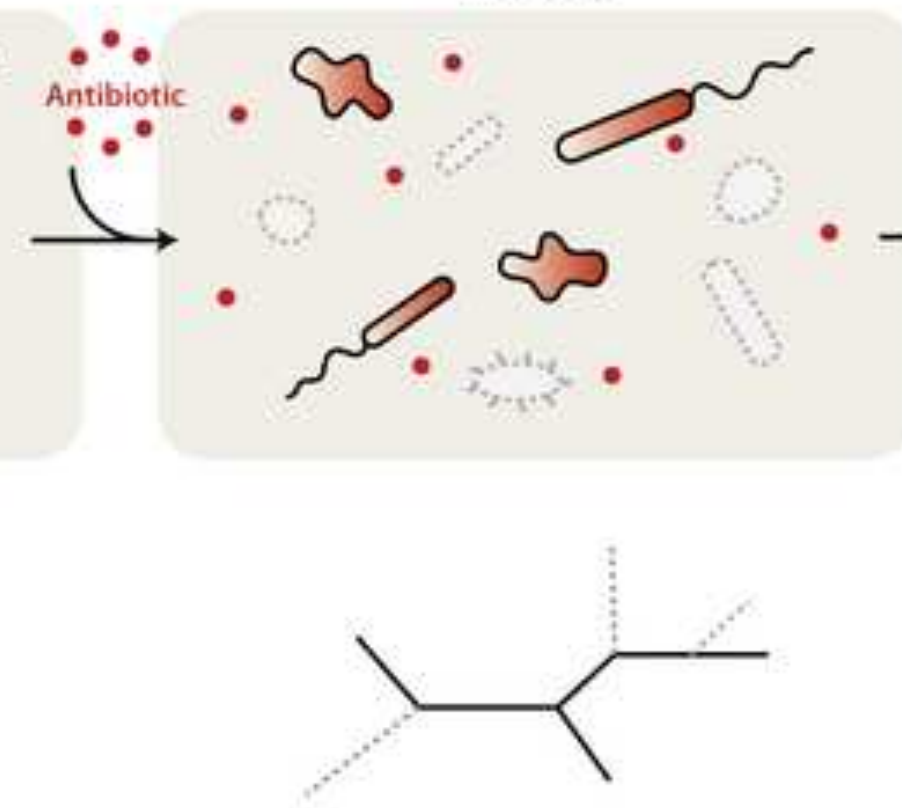

Post exposure

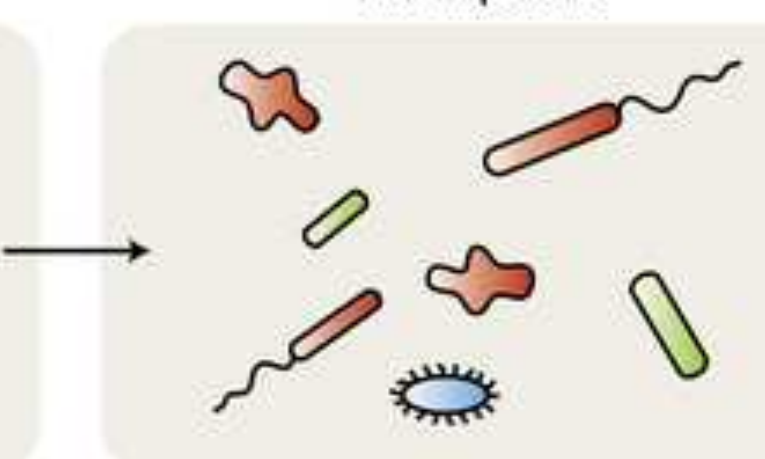

C
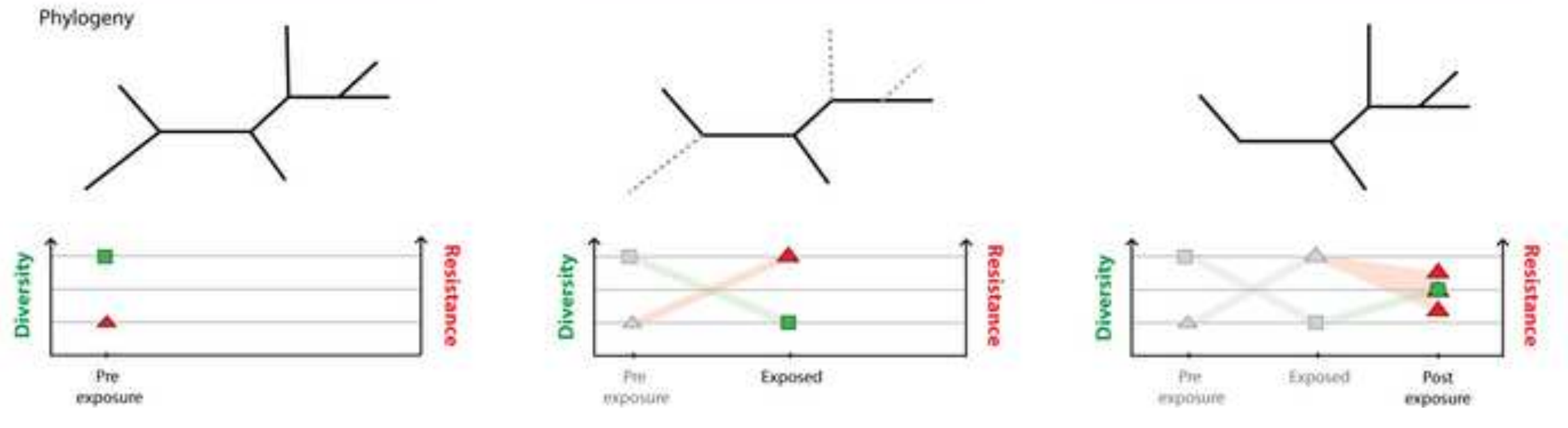


\section{Culture-dependent}

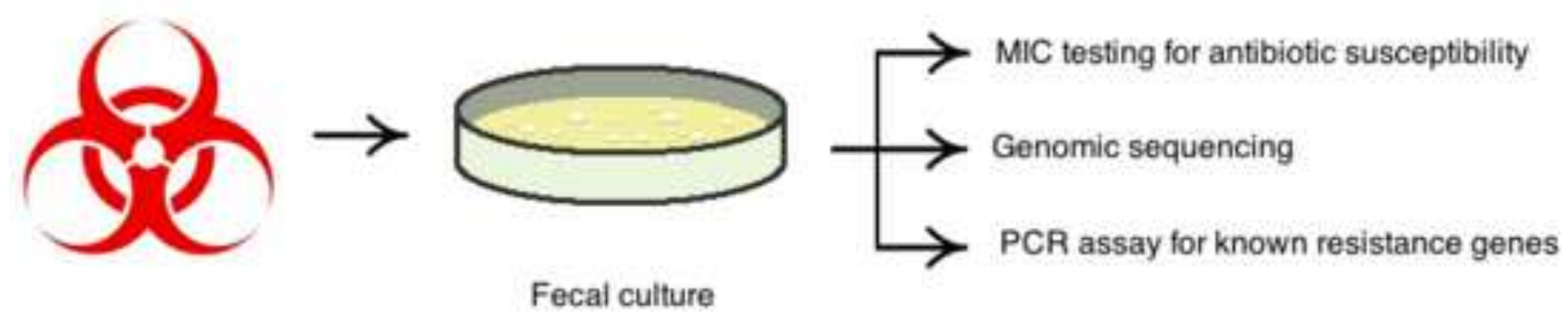

\section{Culture-independent}
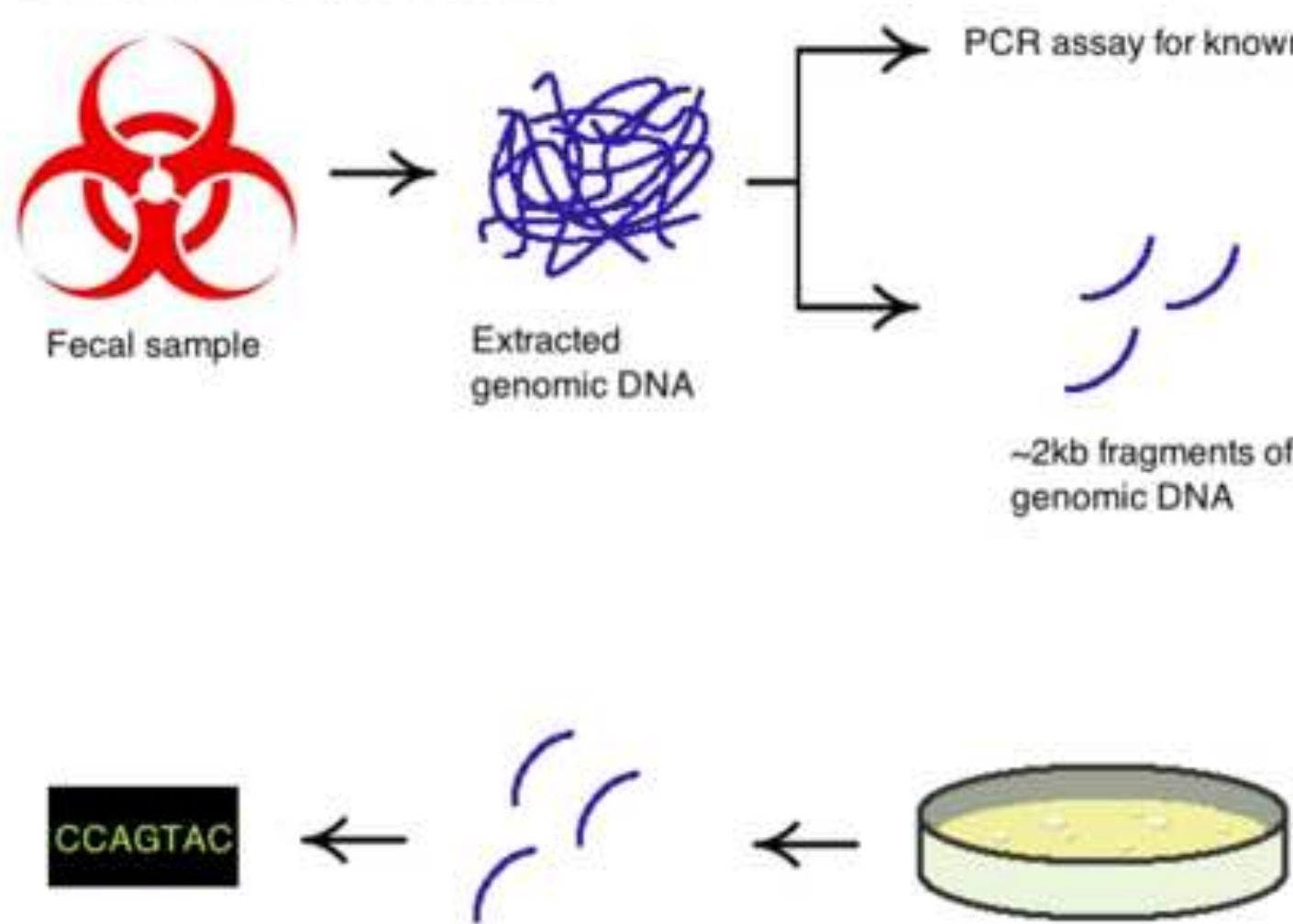

Functional selection on antibiotic-containing growth media
Sequencing and annotation
PCR amplification of selected resistance genes

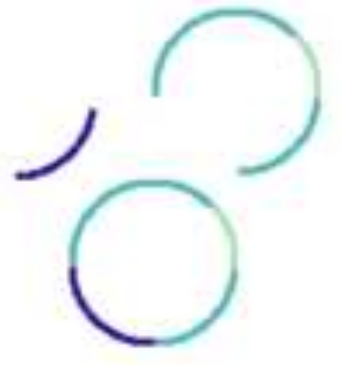

Genomic DNA fragments ligated into plasmid vector

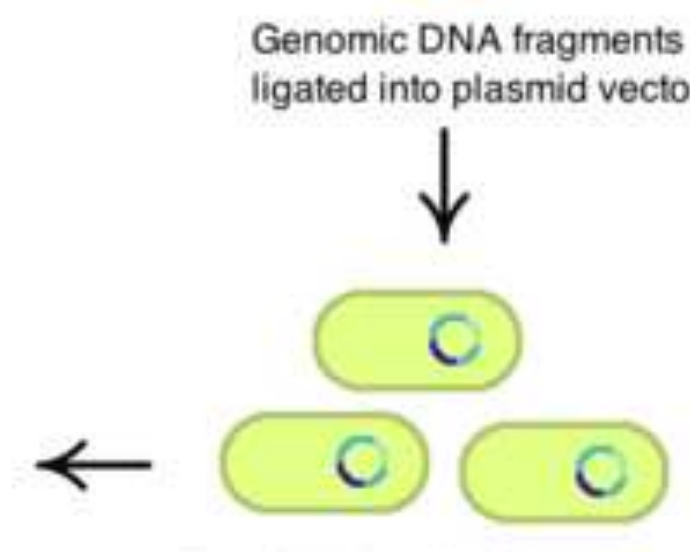

Transfection of indicator host strain (e. g. E. coli)
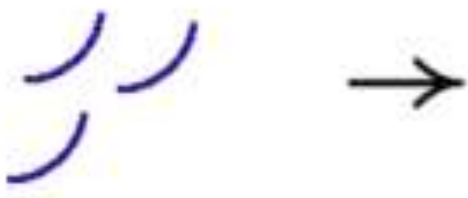